\title{
Physics of psychophysics: Large dynamic range in critical square lattices of spiking neurons
}

\author{
Emilio F. Galera $\mathbb{C}^{*}$ and Osame Kinouchi ${ }^{\dagger}{ }^{\dagger}$ \\ Universidade de São Paulo, FFCLRP, Departamento de Física, Ribeirão Preto, São Paulo 14040-901, Brazil
}

(Received 25 January 2020; accepted 5 June 2020; published 13 July 2020)

\begin{abstract}
Psychophysics tries to relate physical input magnitudes to psychological or neural correlates. Microscopic models to account for macroscopic psychophysical laws, in the sense of statistical physics, are an almost unexplored area. Here we examine a sensory epithelium composed of two connected square lattices of stochastic integrate-and-fire cells. With one square lattice, we obtain a Stevens's law $\rho \propto h^{m}$ with Stevens's exponent $m=0.254$ and a sigmoidal saturation, where $\rho$ is the neuronal network activity and $h$ is the input intensity (external field). We relate Stevens's power-law exponent with the field critical exponent as $m=1 / \delta_{h}=\beta / \sigma$. We also show that this system pertains to the directed percolation (DP) universality class (or, perhaps, the compact-DP class). With two stacked layers of square lattices and a fraction of connectivity between the first and second layer, we obtain at the output layer $\rho_{2} \propto h^{m_{2}}$, with $m_{2}=0.08 \approx m^{2}$, which corresponds to a huge dynamic range. This enhancement of the dynamic range only occurs when the layers are close to their critical point.
\end{abstract}

DOI: 10.1103/PhysRevResearch.2.033057

\section{INTRODUCTION}

Psychophysics is perhaps the oldest experimental part of psychology, starting with the pioneering work of Fechner in 1860 [1]. Its main aim it to describe how sensation is related to the input level reaching a sensory organ. Psychophysical laws are emergent properties of neuronal networks [2]. A fundamental problem in these laws is that they relate several orders of magnitude of input to few orders of magnitude of output. This means that biological sensors have a huge dynamic range (DR), which they should have since natural stimuli vary by orders of intensity. For example, several experimental results can be fitted by a Hill curve [3],

$$
S(I)=\frac{S_{\max } c I^{m}}{S_{\max }+c I^{m}},
$$

where $S(I)$ is the sensation level (to be measured in some scale, $S_{\max }$ is a saturation level, $c$ is a constant, and $I$ is the input level). For moderate input such that $c I^{m} \ll S_{\max }$, we have the famous Stevens's power law [2-7]:

$$
S(I)=c I^{m},
$$

where $m$ is the so-called Stevens's psychophysical exponent. If $m<1$, we have a compressive curve with a large dynamic range.

\footnotetext{
*emiliofgalera@gmail.com

†osame@ffclrp.usp.br
}

Published by the American Physical Society under the terms of the Creative Commons Attribution 4.0 International license. Further distribution of this work must maintain attribution to the author(s) and the published article's title, journal citation, and DOI.
It is not clear how to get this result because sensory neurons at the periphery have a small dynamic range. In a sense, we have a problem typical of statistical physics: how to construct a microscopic model (here based in neurons) that explains a high-level phenomenological law as a collective phenomenon.

The idea that certain parts of the brain can benefit from operating near the critical point of a continuous phase transition has been around for some time now [8-13]. In particular, it has been shown that criticality enables a network of excitable elements with small DR to present a large dynamic range as a collective property, both at the theoretical [14-23] and experimental $[17,24-26]$ levels.

A result very similar to the Hill curve is predicted by some computational models [14-16], but without a simple analytic form as Eq. (1), suggesting that the use of a Hill curve in psychophysics is only a phenomenological or fitting procedure that cannot be obtained from first principles. This view of a large dynamic range as a collective or emergent property (critical or not) of networks of excitable cells is relatively new [27-30].

The standard textbook model to account for a large DR constructed from small DR units is some variation of recruitment theory: sensory neurons, which present sigmoidal responses with short DRs but different response thresholds, are combined to produce a total output with a large DR. In these models, the value of exponent $m$ is not predicted or constrained (see, as examples, [3,31,32]). Recruitment theory has a major flaw: for a wide range of stimulus to be perceived, an equally wide variety of receptor expression in sensory cells must occur. Experimentally, however, receptor overexpression is only about two- or threefold [31], so it is plausible to assume that this is not the main mechanism responsible for the phenomenon [15]. Also, the model mechanism is somewhat oversimplistic: a simple linear sum of sigmoids with a 
distribution of cells fitted by hand to produce a more or less acceptable power law in some input range.

In a sense, recruitment theory is a curve-fitting exercise with sigmoids, not a predictive theory. For example, it does not predict the possible values for Stevens's exponent $m$ (as will be done here); this exponent is only a fitting parameter which is unable to give deep insight into the underlying neuronal network model. On the other hand, in our theory, $m$ relates to the statistical physics field critical exponent as $m=1 / \delta_{h}$.

Here we offer a modern view about psychophysical laws: any network of excitable cells, indeed any excitable media $[14,16,27]$, produces a Stevens's response $S \propto I^{m}$ for moderate $I$ (and a Hill's-type saturation afterwards). This is an intrinsic and basic collective property of excitable cells. So, we need not devise some tricky mechanism to obtain Stevens's law; it is there from start, as a basic property of any network of excitable cells.

In this paper, we study stochastic integrate-and-fire neurons interacting in two coupled square lattices that would be a toy model for a biological sensor. We assume that the coupling inside the square lattices is done by electric synapses, as observed say in the retina or the olfactory bulb. We show that in each layer, there occurs a continuous phase transition from a silent phase to an active phase. This is called an absorbing phase transition: the absorbing phase corresponds to silence or zero activity, from which the system cannot spontaneously escape. The active phase emerges with a given critical exponent from the critical point. Almost all such transitions pertain to the ubiquitous directed percolation (DP) class $[33,34]$ or, perhaps, the so-called compact DP (C-DP or Manna) class, which, in the square lattice $(d=2)$, has specific critical exponents that define its universality class.

Our $2 d$ result is not surprising since the mean-field DP exponent $m^{\mathrm{MF}}=1 / \delta_{h}=1 / 2$ has already been found for complete graphs and random networks [14,35,36]. However, our $2 d$ DP exponent $m \approx 0.254$ means that $S \propto I^{0.254}$, which is a huge improvement over the mean-field $S \propto I^{0.5}$ result.

The second square lattice layer is put after the first one, with a small fraction $p$ of electrical synapses between them. The aim of constructing such a retinalike two-layered sensor is to show that other Stevens's exponents can be obtained by changing the network topology. This has already been demonstrated for random networks [37], where the second layer presented Stevens's exponent $m_{2}^{\mathrm{MF}}=1 / 4=\left(m^{\mathrm{MF}}\right)^{2}$ (the index in $m_{2}$ refers to the second layer output). Here we obtain a similar result, but with a huge dynamic range given by $m_{2}=0.078 \approx m^{2}$; that is, an input range of $O\left(10^{12}\right)$ (similar to the difference between luminosity at midnight and noon) can be mapped onto an $O(1)$ output.

\section{MODEL AND METHODS}

We use a stochastic leaky integrate-and-fire (LIF) neuron originally proposed by Gerstner and van Hemmen [38], rigorously investigated by Galves and Löcherbach [39] and Ferrari et al. [40], and simplified in Refs. [35,41,42]. Time is discrete and updates are done in parallel. For a discussion about LIF discrete-time models, see Refs. [43-45].
The membrane potential of a neuron situated at the $i$ th line and $j$ th column is given by

$$
\begin{gathered}
V_{i j}[t+1]=\mu V_{i j}[t]+I_{i j}[t]+\frac{1}{4} \sum_{k l \in \mathcal{V}}^{4} W_{i j, k l} X_{k l}[t], \\
\Longleftrightarrow X_{i j}[t]=0, \\
V_{i j}[t+1]=0, \Longleftrightarrow X_{i j}[t]=1 .
\end{gathered}
$$

Here, $X_{i j}[t]$ is a binary state variable $(X=1=$ spike, $X=0=$ silence). A neuron stays in its active state for only one time step, assumed here as the typical spike width of 1 $\mathrm{ms}$. If at a given time $t$ the neuron with indexes $i j$ fires, its membrane potential is reset to zero, given by Eq. (4), and otherwise the neuron will follow Eq. (3). The parameter $\mu \in$ $[0,1]$ is a leakage term which controls how much the neuron remembers from its previous voltage $V_{i j}[t]$, and $I_{i j}[t]$ is the external input. Neurons interact in the two-dimensional lattice where each one is connected to its four nearest neighbors (the von Neumann four-sites neighborhood is $\mathcal{V}$ ). The strength of the (electric) synapse between the postsynaptic neuron $i j$ and the presynaptic neuron $k l$ (with $k=i$ and $l=j \pm 1$, or $k=i \pm 1$ and $l=j$ ) is denoted by $W_{i j, k l}$. We use periodic boundary conditions.

In this stochastic model, the firing of a neuron is probabilistic and given by a firing function $\Phi$ :

$$
P\left(X_{i j}[t]=1 \mid X_{i j}[t-1]=0\right)=\Phi\left(V_{i j}[t]\right) .
$$

Notice that this form emphasizes that the neuron has one time step of absolute refractory period, although this is implicit because we assume $\Phi(V=0)=0$. The function $\Phi$ needs only to be a sigmoidal function. For mathematical convenience, we use the so-called rational function [41,42],

$$
\Phi(V)=\frac{\Gamma(V-\theta)}{1+\Gamma(V-\theta)} \Theta(V-\theta) .
$$

Here, $\theta$ is a firing threshold below which the neuron cannot fire, i.e., $\Phi(V)=0$ for $V<\theta$. The $\Gamma$ parameter in Eq. (6) is the neuronal gain. Notice that both the gain $\Gamma$ and threshold $\theta$ are parameters experimentally related to the phenomenon of firing-rate adaptation [46,47]. Although not implemented here, a homeostatic dynamics in the individual synapses $W_{i j, k l}$ and thresholds $\theta_{i j}$ can be used to self-organize the network towards the critical state [36]. So, in our discussion about maximizing the dynamic range at criticality, we will assume that such homeostatic mechanisms can operate in our network.

For a meticulous analysis of the mean-field approximation regarding this model, we refer to $[35,41,42]$. We will show that the system undergoes an absorbing second-order phase transition if the external field and the firing thresholds are equal, $\theta_{i j}=I_{i j}$; see [36]. This apparent fine-tuning condition will be discussed in Sec. IV. If $\theta_{i j} \neq I_{i j}$, the transition is of first order [35,41].

The activity of a network with $N$ neurons is, at any time,

$$
\rho[t]=\frac{1}{N} \sum_{i j} X_{i j}[t]
$$


and it can be measured by

$$
\rho(w, \Gamma)=\frac{1}{t_{f}-t_{i}} \sum_{t=t_{i}}^{t_{f}} \rho[t],
$$

where $\left(t_{i}, t_{f}\right)$ marks a large time interval in the simulation far from transient states. The time average $\rho(W, \Gamma)$, given by Eq. (8), is used as our order parameter, and the average synaptic weight $W=\left\langle W_{i j, k l}\right\rangle$ and average gain $\Gamma=\left\langle\Gamma_{i j}\right\rangle$ are our control parameters. We notice that if at a given time step we have $\rho[t]=0$, then a random site is chosen and its state is put as $X_{i}[t+1]=1$. This is done to get the network out of the absorbing state.

We define another quantity, the order parameter fluctuations $\Delta \rho$, related to the fluctuations of the activity $\rho$,

$$
\Delta \rho=N\left(\left\langle\rho^{2}\right\rangle-\langle\rho\rangle^{2}\right) .
$$

In order to evaluate how the neuron model responds to external stimulus when interacting in a layered system, one needs to know how a single $2 d$ lattice of neurons works. First, with $\Gamma=1$ fixed, we explore the effect of the control parameter $W$ to roughly determine where the phase transition occurs, i.e., we need to know where the subcritical, critical, and supercritical regimes are. Then, we can refine our measurements and explore the critical region for systems with different sizes $N$ and use finite-size scaling techniques [48] to better determine the critical point of the transition $W_{c}$, the order parameter critical exponent $\beta$, and the susceptibility critical exponent $\gamma^{\prime}$.

In the vicinity of the critical point, $\rho$ and $\Delta \rho$ should scale as

$$
\rho \propto|\bar{W}|^{\beta}, \Delta \rho \propto|\bar{W}|^{-\gamma^{\prime}},
$$

where $\bar{W}$ is the reduced control parameter $\bar{W}=(W-$ $\left.W_{c}\right) / W_{c}$.

We then proceed to our bottom-up psychophysics approach, where we build a sensor with two layers of neurons. Each layer is a square lattice. The first one is stimulated by the external stimulus, modeled as a Poisson process. The probability per time step of a neuron being activated is

$$
\lambda=1-e^{-r} .
$$

Here, $r$ is a stimulation rate. Neurons in the first layer can either fire due to synaptic excitation from neighbors or with probability $\lambda$ due to the input Poisson process.

On the second layer, we randomly chose $p=0.1 \mathrm{~N}$ neurons to connect to the first layer. These neurons receive an input $I_{i j}[t]=J X_{i j}^{1}[t]$, where $X_{i j}^{1}$ is the neuron of the first layer; that is, both connected neurons of the first and second layer share the same indexes $i j$. The value $J$ used was high enough to guarantee that the connection between layers always pass information; that is, if a neuron in the first layer spikes, then the neuron connected to it in the second layer also forcibly spikes. The electric synapses $J$ are unidirectional; that is, activity in the second layer does not excite back the first layer.

We ran simulations for various system sizes in the three regimes, always stimulating neurons from the first layer with rates ranging from $r=10^{-6}$ up to $r=0.1$. We will show that in the critical region, the activity $\rho$ in each layer can map stimulation rates $r$ with large dynamic range. For the first layer,

$$
\rho_{1}(r) \propto r^{m}
$$

and for the second layer,

$$
\rho_{2}(r) \propto\left[\rho_{1}(r)\right]^{m} \propto r^{m^{2}} .
$$

Critical systems in the presence of an external field $h$ have a well-established behavior. For a small field, the order parameter scales as a power law,

$$
\rho(h) \propto h^{\beta / \sigma},
$$

where $\beta$ is the order parameter critical exponent and $\sigma$ is the critical exponent associated with the mean cluster size. If we identify the stimulation rate $r$ as the external field $h$, it is possible to write Stevens's exponent $m$ of Eq. (12) as

$$
m=\frac{\beta}{\sigma} .
$$

Equation (14) is valid for asymptotically small fields, $h \rightarrow$ 0 . This means that the relation between exponents (15) is valid as long as the stimulation rate $r$ of the Poisson process (11) is small.

To quantify how each layer responds to the stimulation, we follow the dynamic range definition of Kinouchi and Copelli [14]. We measure the individual activity of each layer as a function of the stimulus rate $r$. We then calculate the dynamic range $\Delta_{h}$ of each layer for the three regimes:

$$
\Delta_{h}=10 \log _{10}\left(\frac{r_{0.9}}{r_{0.1}}\right)
$$

where the values $r_{0.9}$ and $r_{0.1}$ are the stimulation rates which evoke the activities $\rho_{0.9}$ and $\rho_{0.1}$. These activity values are obtained through the equation $\rho_{x}=\rho_{0}+x\left(\rho_{\max }-\rho_{0}\right)$, where the values $\rho_{\max }$ and $\rho_{0}$ are just the largest and smallest (not necessarily zero, due to self-sustained supercritical activity) response of a layer. The dynamic range is a measure that relates, in decibels, the largest and smallest inputs that the system can map in the output.

\section{RESULTS}

\section{A. The $2 d$ lattice with leakage $\mu=0$}

For the $2 d$ network, we first present curves $\rho(W ; N)$ for different square and rectangular lattices (from here, we fix $\Gamma=1$ without loss of generality); see Fig. 1(a).

The solid line has the form

$$
\rho(W ; N \rightarrow \infty) \propto \bar{W}^{\beta},
$$

with the $2 d$ critical point $W_{c}=1.74$. We can see that this curve produces a very good fit of the data for large $N$ if we use the tabulated $2 d$ DP critical exponent $\beta=0.583$ [49]. We also plot the fluctuations $\Delta \rho(W ; N)$ as a function of the control parameter $W$ in Fig. 1(b). We obtain a good fit $\Delta \rho \propto \bar{W}^{-\gamma^{\prime}}$ for large networks if we use the $2 d$ DP tabulated exponent $\gamma^{\prime}=0.2998$ [49].

The significance of the DP transition for our neuronal network model is the following. The model admits a silent phase $(\rho=0)$ but, with increasing coupling, there occurs a change of phases. This could be an oscillatory phase or a 


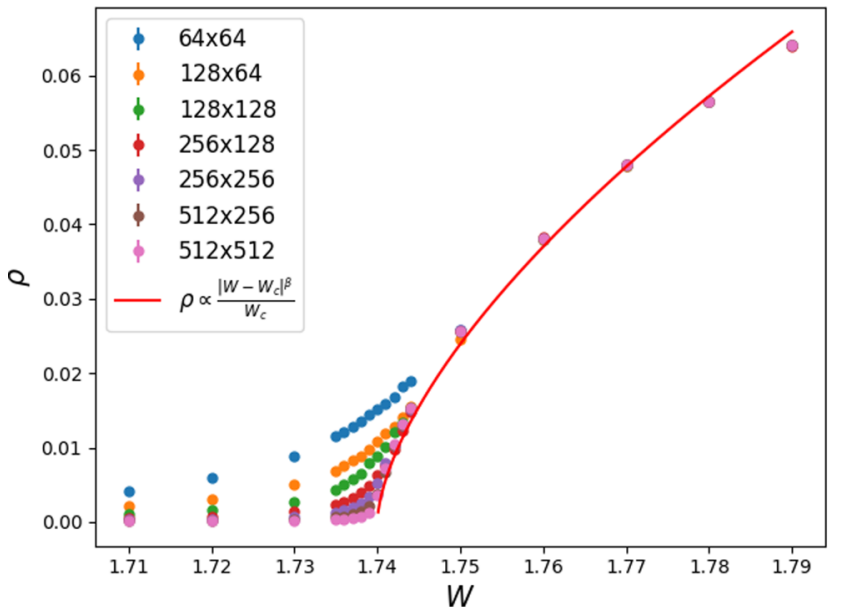

(a)

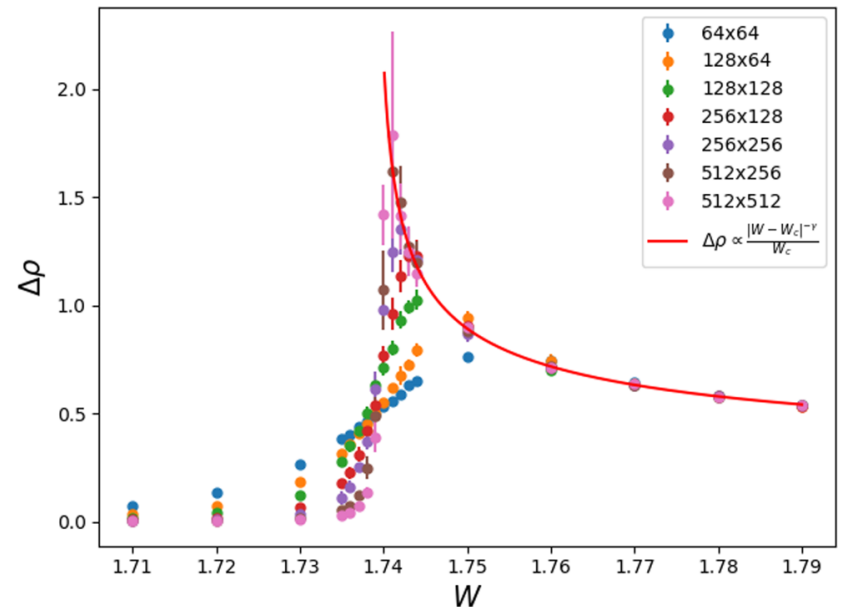

(b)

FIG. 1. (a) Order parameter $\rho(W ; N)$ for systems of different sizes close to the critical region. (b) Order parameter fluctuations $\Delta \rho(W ; N)$ for various system sizes near the critical region. For growing lattices, the data approximates the mean-field result (solid line).

coexistence with two fixed points for the same coupling (a nontrivial $\rho$ phase and the trivial $\rho^{0}$ absorbing phase). This last case is achieved by a discontinuous (first-order) phase transition.

In our case, we found a continuous (second-order) transition from the absorbing state $\rho^{0}$ to an active phase $\rho$ at a critical point $W_{c}$. As discussed in Sec. II, critical exponents can be defined only for continuous transitions. The found exponents enable us to classify our transition as pertaining to the directed percolation (DP) universality class, or perhaps the compact DP (Manna) class. This is not so surprising because almost all continuous transitions from a single absorbing state pertain to such classes and follow the so-called Janssen-Grassberger conjecture [33,34]. What perhaps is a bit curious is that such a conjecture works in a model with somewhat complicated elements such as our stochastic LIF neurons.

We tried, however, to obtain the critical exponents in an independent way. In the critical region, both $\rho_{N}$ and $\Delta \rho_{N}$ are strongly dependent on the system size $N$. To get around the fact that we are far from the thermodynamic limit, we use finite-size scaling techniques. First, we rescale $\rho$ and $\Delta \rho$ :

$$
\begin{gathered}
\rho(\bar{W} ; L)=L^{-\beta / \nu_{\perp}} G_{\rho}\left(L^{1 / v_{\perp}}|\bar{W}|\right), \\
\Delta \rho(\bar{W} ; L)=L^{\gamma^{\prime} / v_{\perp}} G_{\Delta \rho}\left(L^{1 / v_{\perp}}|\bar{W}|\right),
\end{gathered}
$$

where $L=\sqrt{N}$ is the characteristic square network size, $v_{\perp}$ is the spatial correlation length critical exponent, and $G_{\rho}$ and $G_{\Delta \rho}$ are scaling functions. Then, we plot $\rho$ and $\Delta \rho$ as functions of the characteristic system size $L$ for different values of $W$. When $W=W_{c}$, the reduced control parameter is $\bar{W}=0$. The rescaled parameters $\rho$ and $\Delta \rho$ are then power laws which depend only on $L$,

$$
\begin{gathered}
\rho(0 ; L)=L^{-\beta / \nu_{\perp}} G_{\rho}(0), \\
\Delta \rho(0 ; L)=L^{\gamma^{\prime} / \nu_{\perp}} G_{\Delta \rho}(0) .
\end{gathered}
$$

The fine determination of $W_{c}, \beta, \gamma^{\prime}$, and $v_{\perp}$ can be done with standard finite-size techniques, as delineated above.
However, this is not our main concern here since that would require much more computational effort and is not the main subject of the paper. By now, it is sufficient to show that the critical exponents of our stochastic integrate-and-fire neuronal network are compatible with the $2 d$ DP class or with the $2 d$ compact-DP class (Manna class); see Table I.

First, simulation results for large $N$, varying $W$, indicate $W_{c} \rightarrow 1.74 \pm 0.01$. Then, a simple fit of the data in Fig. 1(a) to Eq. (10) yields $\beta \approx 0.564 \pm 0.05$. By using Eqs. (20) and Fig. 2 fits, we obtain $\beta / v_{\perp} \approx 0.77$ and $\gamma^{\prime} / \nu_{\perp}=0.50$, that is, $\nu_{\perp} \approx 0.73 \pm 0.06$ and $\gamma^{\prime} \approx 0.37 \pm 0.04$; see Table I. We also have obtained, from the dependence on the external field at the critical point, $m=1 / \delta_{h}=0.254 \pm 0.005$; see Sec. III C. From the equality $\delta_{h}=\sigma / \beta$ [49], we obtain $\sigma=2.22 \pm 0.05$. We observe that the errors are not statistical, but simple fitting errors; see Table I.

We also present a data collapse by plotting curves $L^{\beta / v} \rho \times$ $L^{1 / v}|\bar{W}|$ and $L^{-\gamma / v} \Delta \rho \times L^{1 / \nu}|\bar{W}|$; see Fig. 3. Beside showing an agreement of the exponents, the collapse gives the scaling functions $G_{\rho}$ and $G_{\Delta \rho}$. The obtained critical exponents, the data collapse of the $\rho_{N}(\bar{W})$ and $\Delta \rho_{N}(\bar{W})$ curves, and the Janssen-Grassberger conjecture $[33,34]$ seem to be enough reasons to consider that our stochastic leaky integrate-and-fire neuronal network indeed belongs to the DP or the C-DP universality classes (the exponents of these two classes are

TABLE I. Critical exponents (directed percolation and Manna exponents from [49]).

\begin{tabular}{lccc}
\hline \hline \multicolumn{3}{c}{$2 d$ exponent } \\
\hline & Results & DP & Manna \\
\hline$\beta$ & $0.56 \pm 0.05$ & $0.5834 \pm 0.0030$ & $0.624 \pm 0.029$ \\
$\nu_{\perp}$ & $0.73 \pm 0.05$ & $0.7333 \pm 0.0075$ & $0.799 \pm 0.014$ \\
$\gamma^{\prime}$ & $0.37 \pm 0.05$ & $0.2998 \pm 0.0162$ & $0.367 \pm 0.019$ \\
$m=1 / \delta_{h}$ & $0.25 \pm 0.05$ & $0.268 \pm 0.001$ & $0.280 \pm 0.014$ \\
$\sigma=\beta / m$ & $2.29 \pm 0.05$ & $2.1782 \pm 0.0171$ & $2.229 \pm 0.032$ \\
\hline \hline
\end{tabular}




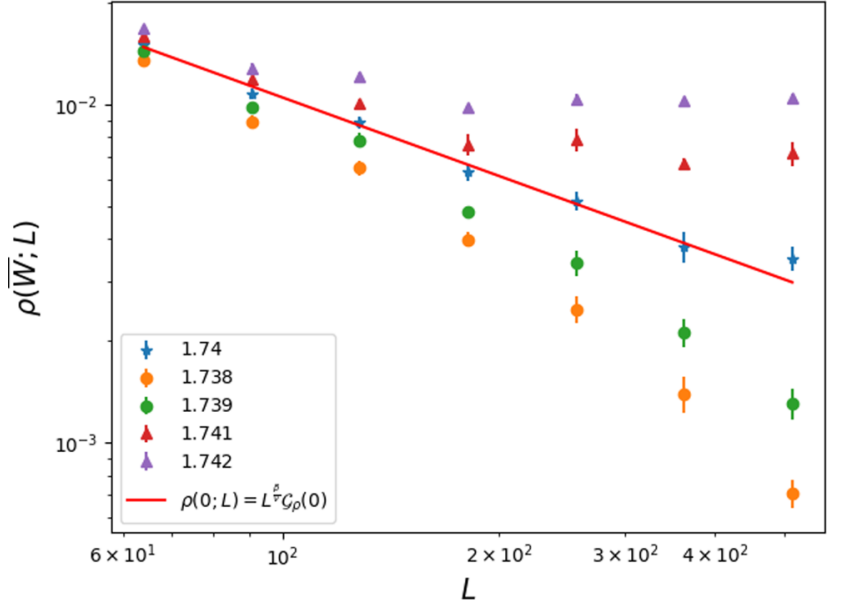

(a)

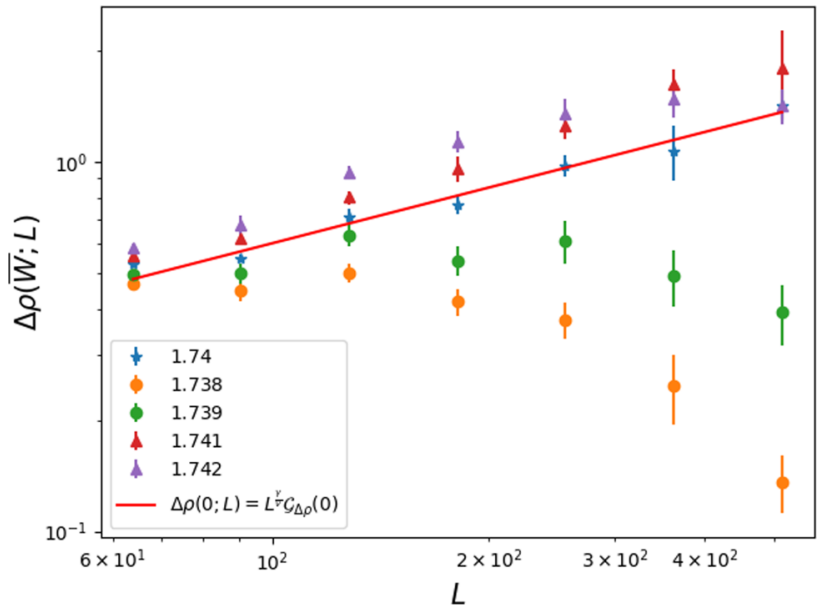

(b)

FIG. 2. (a) Order parameter $\rho$ and (b) $\Delta \rho$ as a function of $L$. Inverted triangles mark the critical point $W_{c}=1.74$. Red lines are curve fits to measure the exponent ratios of Eq. (20): $\beta / \nu_{\perp} \approx 0.77$ and $\gamma^{\prime} / \nu_{\perp} \approx 0.50$.

very similar and it is difficult to determine the class only from the numerical results; see Table I).

\section{B. The case with leakage $\mu>0$}

The leakage parameter $\mu$ in Eq. (3) is the ingredient that makes our neuron different from a simple binary automaton and to be defined as an integrate-and-fire element. The neuron has memory of its previous inputs because it forgets its membrane potential with a timescale given by $\mu$.

We found that $\mu>0$ changes the location of $W_{c}$ [Fig. 4(a)]. In the mean-field case, we obtain analytically that $W_{c}(\mu)=$ $(1-\mu) W_{c}(0)$, with $W_{c}(0)=1($ for $\Gamma=1)[35,42]$ :

$$
\rho(\mu)=\left[C(\mu) \frac{W-W_{c}(\mu)}{W_{c}(\mu)}\right]^{\beta},
$$

where $C(\mu)$ is independent of $W$. This means that if $x=$ $W-W_{c}(\mu)$, the function $y(x)=\left[W_{c}(\mu) / C(\mu)\right]^{\beta} \rho(\mu)$ is independent of $\mu$.

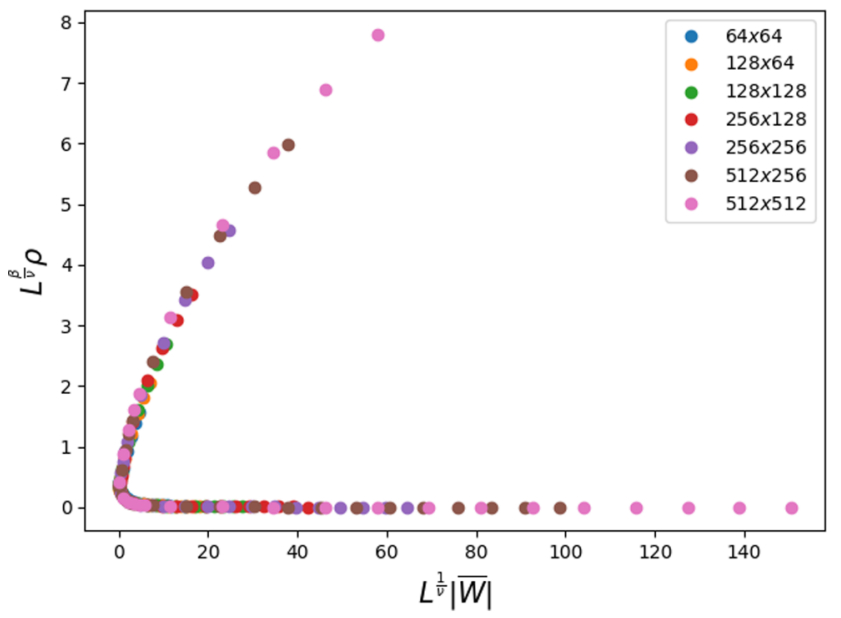

(a)
We searched for a similar result for our square lattice, but now with $W_{c}(\mu)=(1-\mu) W_{c}(0)=(1-\mu) 1.74$. We find that the collapse is not good (not shown), meaning that the mean-field result $W_{c}(\mu)=(1-\mu) W_{c}(0)$ does not generalizes to the $2 d$ case. However, by using the measured $2 d W_{c}(\mu)$ from Fig. 4(a), we obtain a very good data collapse; see Fig. 4(b). This collapsed data means that leakage $\mu>0$ does not change the universality class of our system.

\section{Dynamic range of the first layer}

As can be seen in Fig. 5(a), the value of the exponent $m \approx 0.254 \pm 0.005$ is close to the expected value $m=\beta / \sigma=$ $0.268 \pm 0.001$, if we use the DP class tabulated values for $\beta$ and $\sigma$. Assis and Copelli [16] found comparable values for the Stevens's exponent $m$ for a susceptible-infected-recoveredsusceptible (SIRS) model in the square lattice.

As already observed, from our data, we obtain $\sigma=$ $\beta / m=0.63 / 0.254=2.48 \pm 0.05$. By using the tabulated $\beta=0.5834$, we get a better value, $\sigma=2.29 \pm 0.01$, to be compared with the DP value, $\sigma=2.1782 \pm 0.0171$ [49].

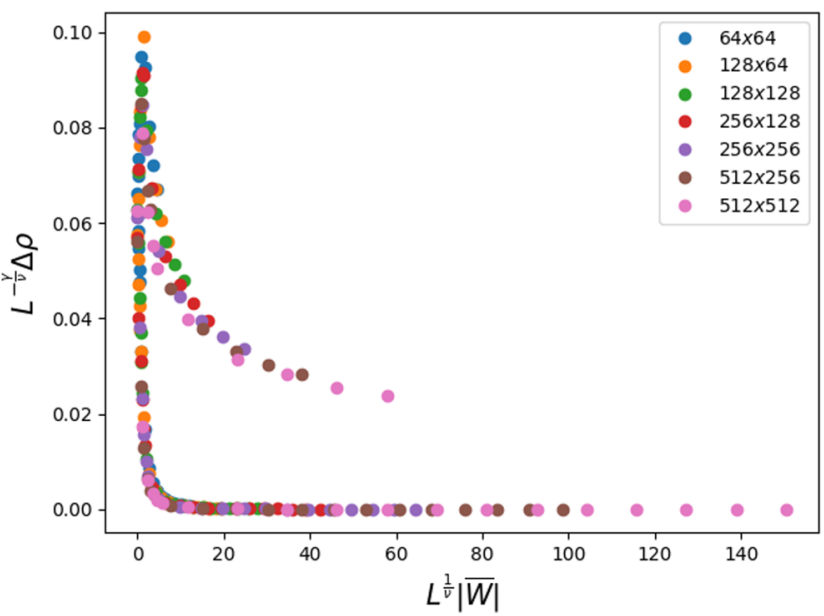

(b)

FIG. 3. (a) Data collapse for the order parameter $L^{\beta / \nu_{\perp}} \rho(\bar{W})$ and (b) fluctuations $L^{-\gamma^{\prime} / \nu_{\perp}} \Delta \rho$. 


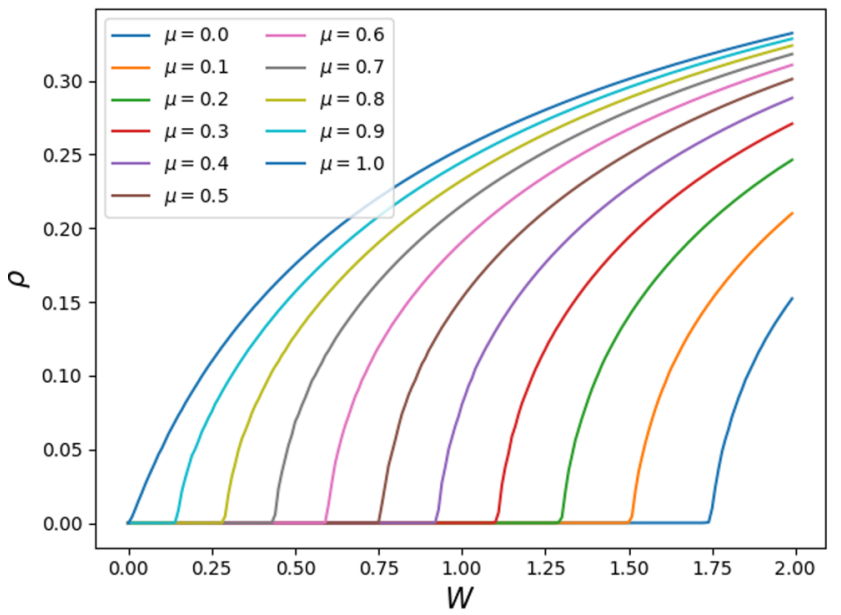

(a)

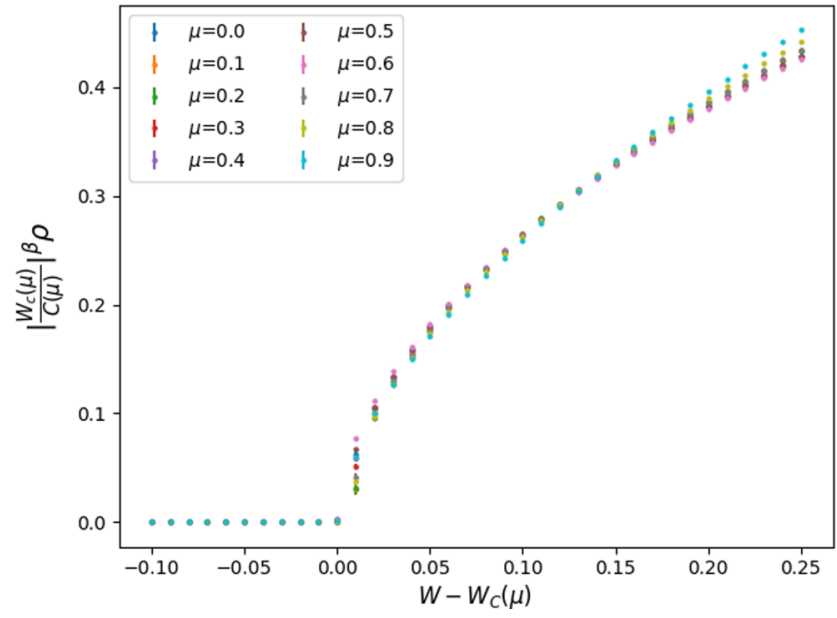

(b)

FIG. 4. (a) Curves $\rho(W, \mu)$ for increasing $\mu$ from right to left and $N=512 \times 512$; (b) data collapse using $x=W-W_{c}(\mu)$ and $y=$ $\left[W_{c}(\mu) / C(\mu)\right]^{\beta} \rho(\mu)$.

To understand how much the regimes influence the response, consider Fig. 5(a). In the subcritical regime, since the coupling between neurons is small, the external input does not propagate and the system response is linear. The same thing happens in the supercritical regime, but for a different reason: in this case, we have self-sustained activity and small inputs are lost in a noisy environment. It is only close to criticality (a few percent from $W_{c}$ ) that both small and large stimulus alike can be mapped in the output [14,16-21,24]. The dynamic range $\Delta_{h}$ of the first layer can be seem in Fig. 5(b). At criticality, one can obtain a $\Delta_{h} \approx 32 \mathrm{~dB}$ for $L=256$, and this value can be higher for larger networks.

\section{Dynamic range of the second layer}

Like the case of the first layer, we present examples of the response function $\rho_{2}$ in the three regimes; see Fig. 6(a). The fit for the critical power law gives $m_{2} \approx 0.078$ (the index refers to the second layer). This accords with the expected value

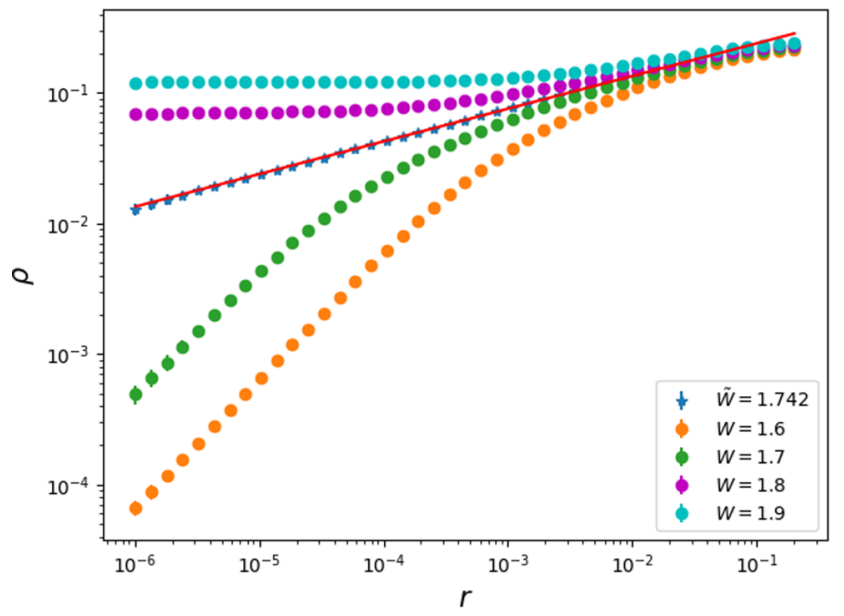

(a) for the exponent of Eq. (13), which is $m_{2}=m^{2}=0.072$, if we assume that the first layer represents the external input for the second layer. Here, a fraction $p=0.1$ of the neurons of the first layer is connected randomly to the second layer by forcing synapses; that is, if the corresponding first neuron spikes, the connected neuron in the second neuron spikes after a time step.

The dynamic range of the second layer in the subcritical and supercritical regime is low for the same reason that they are small in the first layer. However, in the critical regime, the dynamic range of the second layer is huge [above 40 dB; see Fig. 6(b)] and sufficient to account for the exquisite performance of biological sensors.

\section{E. The effect of the interlayer connectivity $p$}

As observed, for small interlayer connectivity $p=0.1$, the second layer exponent seems to preserve the relation $m_{2}=0.072 \approx m^{2}=\left(1 / \delta_{h}\right)^{2}[48,50]$. However, for larger $p$,

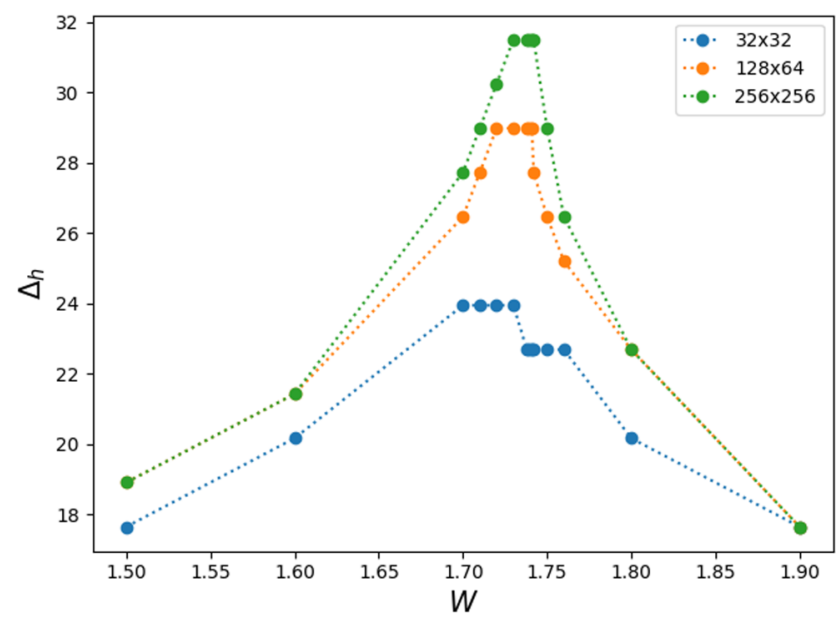

(b)

FIG. 5. (a) First layer activity $\rho(r, W)$ as a function of stimulation rate $r$. The red line $\left(W_{c}=1.742\right)$ is the power law with exponent $m=0.254$. Network size $N=256 \times 256$. (b) Dynamic range for the first layer, for several values of $W$ and systems with increasing sizes from bottom to up. 


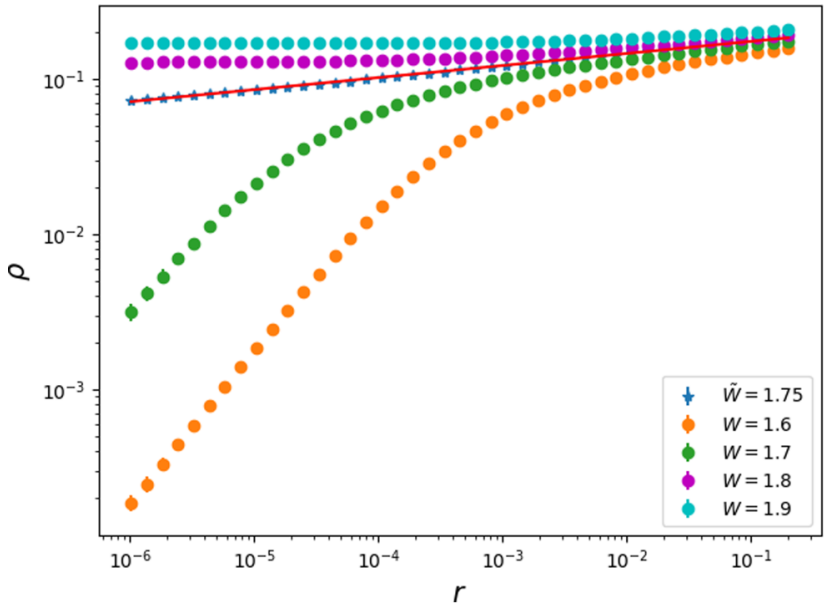

(a)

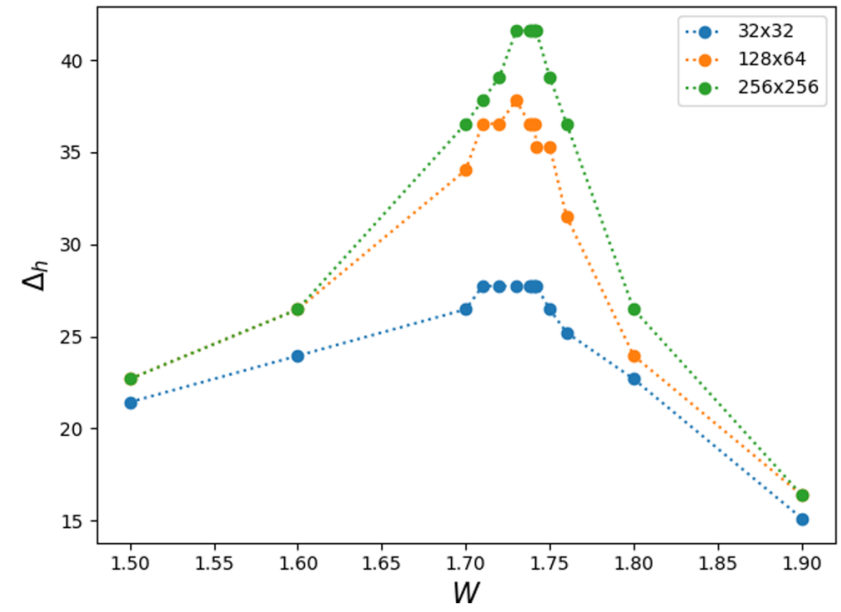

(b)

FIG. 6. (a) Second layer neuronal activity $\rho_{2}(r, W)$ as a function of stimulation rate $r$ at the first layer. The red line $\left(W_{c}=1.75\right)$ refers to the power law given by Eq. (13) with $m_{2}=0.078$. Network size $N=256 \times 256$. (b) Dynamic range for the second layer, for several values of $W$ and increasing sizes from bottom to up (the first layer has $N=256 \times 256$ neurons).

we deviate from this behavior; see Fig. 7 for simulations with $0.05 \leqslant p \leqslant 0.5$.

We must remember here the origin of the increase of dynamic range in networks of excitable cells (in contrast to pools of isolated cells in recruitment theory). Networks enable signal amplification, that is, the stimulation of one cell by an input signal can produce a cascade of firings in neighbors (a branching process). This branching of the original signal means that small signals are amplified, increasing the response to them. On the other hand, the saturation due to very large input is delayed because the branching processes interact and, since the cells have refractory periods, the activity is suppressed [27-29]. This occurs both for subcritical and supercritical networks (their DR is always better than a pool with the same number of isolated neurons), but the optimal point is the critical one $[14,15]$.

A very low $p$ means that the layers are uncoupled, so the signal amplification mechanism does not work. On the other hand, a large connection $p$ means that the activity of the first layer, which is already increased, is heavily communicated to the second layer. Each neuron that receives a synapse is now the source of a new branching process. This means that high $p$ induces saturation in the second layer, increasing its Stevens's exponent $m_{2}$.

Indeed, from Fig. 7(a), it is possible to see that $m_{2}(p)$ is a monotonically growing function of the connectivity $p$. This variation, however, is not so large $\left(0.07<m_{2}<0.10\right)$; see Fig. 7 inset. From Fig. 7(b), we see that the dynamic range for various $p$ is not sensitive to such small variation.

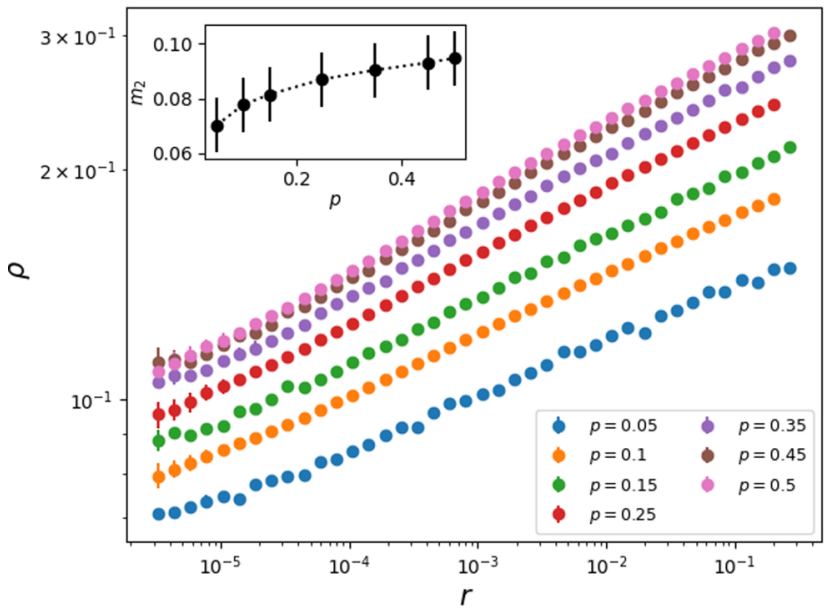

(a)

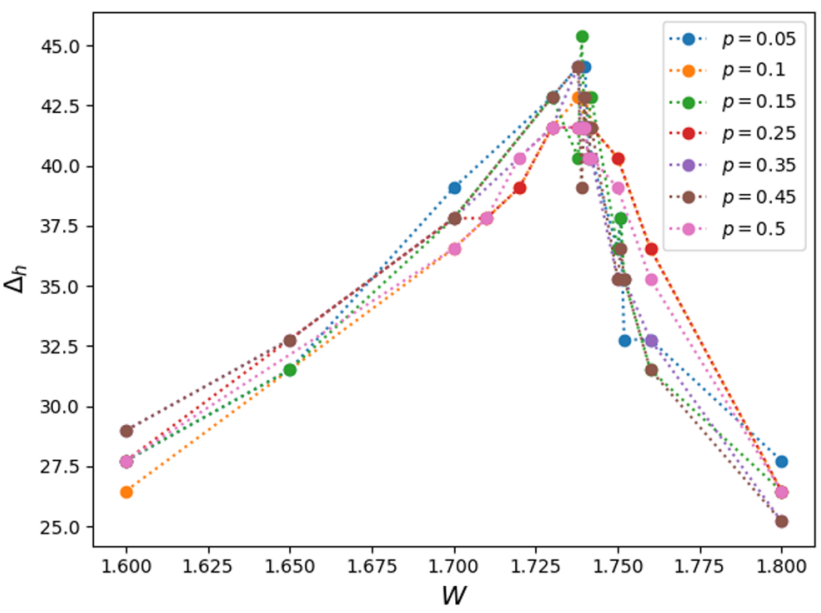

(b)

FIG. 7. (a) Stimulus and response curves for various values of interlayer connectivity fractions $p$ that increases from bottom to up. The inset presents the exponent of the power law $\rho \propto r^{m_{2}}$ as a function of $p$. Simulation was carried using a $128 \times 128$ bidimensional network for each layer and fixed synaptic weight close to the critical point $W=1.75$. (b) Dynamic range for various values of $p$ for the second layer of a bilayered system, where each one consists of a $128 \times 128$ two-dimensional network. 


\section{HOMEOSTATIC CRITICALITY}

Up to now, we have shown that critical networks have maximal dynamic range. However, we have not discussed how biological neuronal networks could tune themselves towards criticality. In a series of publications [22,35,36,41,42,51], we have explored such homeostatic mechanisms, which implement the so-called self-organized quasi-criticality (SOqC) scenario [52,53].

Homeostatic criticality means that the critical point turns out an attractor for some adaptive dynamics of the system. In contrast to conservative SOC models such as sand piles, where the self-organization depends on dissipation on the borders of the system that have no explicit equations for that, $\mathrm{SOqC}$ models such as forest-fire models or neuronal networks have explicit drive and dissipation mechanisms.

For example, let us consider activity-dependent synapses $[22,36,54]$ such that they depress by a factor $u$ when the presynaptic neuron fires (due to vesicle depletion) and recover toward a baseline level $A$ with a characteristic time $\tau$ :

$$
\begin{aligned}
W_{i j, k l}[t+1]= & W_{i j, k l}[t+1]+\frac{1}{\tau}\left(A-W_{i j, k l}[t]\right) \\
& -u W_{i j, k l}[t] X_{k l}[t] \quad(k \in \mathcal{V}),
\end{aligned}
$$

where $k, l$ are the site neighbors of neuron $i, j$. Here, the drive is the recovering mechanism and the dissipation is due to the short-term depression. It is possible to show that with this dynamics and for large $\tau$, the average value $W[t]=\left\langle W_{i j, k l}[t]\right\rangle$ goes towards $W_{c}$.

We have noticed before that the second-order phase transition only occurs when $\theta=I$, where $\theta=\left\langle\theta_{i j}\right\rangle$ and $I=\left\langle I_{i j}\right\rangle$ are the average threshold and average input. This condition, for neuronal networks, seems to be a fine tuning. However, if we think that the field $h=I-\theta$ is the average suprathreshold current, the condition of zero field $h=0$ is a natural requisite for continuous phase transitions in statistical physics.

So, inspired in firing-rate adaptation mechanisms that postulate dynamic thresholds [46], we propose the following homeostatic dynamics:

$$
\theta_{i j}[t+1]=\theta_{i j}[t]-\frac{1}{\tau_{\theta}} \theta_{i j}[t]+u_{\theta} \theta_{i j}[t] X_{i j}[t],
$$

where now the signs are inverted, and therefore the dissipation is due to the $1 / \tau$ decay and the drive (growth of the threshold) occurs when the neuron spikes. It is also possible to show that this adaptive dynamics leads to $\theta[t]=\left\langle\theta_{i j}[t]\right\rangle \rightarrow I$, that is, $h \rightarrow 0$. An experimental prediction of this mechanism is that in critical neuronal networks with power-law avalanches, the neurons mostly adapt their firing thresholds to their external inputs.

So, with these two homeostatic mechanisms, the critical point $W=W_{c}, h=h_{c}=0$ in the phase diagram of the systems turns out an attractor of the overall dynamics. Simulations of these homeostatic mechanisms in the $2 d$ lattice are somewhat out of the scope of this paper, but full results will be presented in future work.

\section{DISCUSSION AND CONCLUSION}

The biological motivation for our the model is the retina, where both lateral and vertical coupling by electric synapses (gap junctions) occur, forming neuronal networks with stacked layers [55]. All these electric synapses are plastic, from the millisecond to the minute timescales [56], which opens the possibility of homeostatic tuning to criticality $[36,42]$, as supposed here. Moreover, there is experimental [57,58] and computational [59] evidence that disruption of electric synapses diminishes the sensitivity and degrades the retina dynamic range. We emphasize that we worked here with stochastic integrate-and-fire neurons, not cellular automata as done in [14-16,22,28,30,37], generalizing thus these results to biologically more realistic elements.

By studying the critical exponents at the second-order phase transition, we found that $2 d$ lattices of stochastic integrate-and-fire neurons are compatible with the directed percolation universality class. We then proposed the topology of two coupled square lattices to increase the dynamic range of a retinalike sensor. The first one receives Poisson inputs at rate $r$, and represents it as a neuronal activity $\rho_{1} \propto r^{m}$, with $m=1 / \delta_{h}=\beta / \sigma=0.268$. This activity is passed, by a fraction $p$ of neurons, to the second layer which then presents an output activity $\rho_{2} \approx r^{m_{2}}$. The final Stevens's exponent of the system is $m_{2}=0.078 \approx m^{2}=(\beta / \sigma)^{2}=0.072$. Thus, the exponent relation given by Eq. (15) proposed in [14] seems to be valid, regardless of topology, as long as the stimulus intensity is moderate: the power-law response is valid only before a saturating regime (Hill's-like curve) also found in biological sensors.

It is possible to show that $1 d$ systems (a ring of neurons) pertain to the $1 d$ DP class $[16,60]$ (or perhaps the $1 d$ Manna class). In this case, we have a very large dynamic range due to the expected value $m=\beta / \sigma=0.276486 / 2.554216=$ 0.108247 . This means that an input range of $O\left(10^{15}\right)$ units can be mapped to an output range of $O(100)$. Although such low-dimensional topologies perhaps have no applications in biology, it is conceivable that artificial sensors with a huge dynamic range could be constructed based in these principles.

\section{ACKNOWLEDGMENTS}

E.F.G. thanks CAPES (Grant No. 142118/2020-6) for financial support. O.K. acknowledges CNAIPS-USP support and FAPESP Scholarship No. BPE 2019/12746-3. This work was produced as part of the activity of FAPESP Research, Innovation and Dissemination Center for Neuromathematics (Grant No. 2013/07699-0, São Paulo Research Foundation). The present work was also realized with the support of $\mathrm{CNPq}$ (Grant No. 309704/2018-9), Conselho Nacional de Desenvolvimento Cientfico e Tecnolgico - Brasil.
[1] G. T. Fechner, Elemente der Psychophysik (Breitkopf and Härtel, Leipzig, 1860).

[2] S. S. Stevens, Psychophysics: Introduction to its Perceptual, Neural and Social Prospects (Wiley, Oxford, UK, 1975).
[3] M. Chastrette, T. Thomas-Danguin, and E. Rallet, Modeling the human olfactory stimulus-response function, Chem. Senses $\mathbf{2 3}$, 181 (1998).

[4] S. S. Stevens, On the psychophysical law, Psychol. Rev. 64, 153 (1957). 
[5] C. T. Kello, G. D. A. Brown, R. Ferrer-i Cancho, J. G. Holden, K. Linkenkaer-Hansen, T. Rhodes, and G. C. Van Orden, Scaling laws in cognitive sciences, Trends Cogn. Sci. 14, 223 (2010).

[6] R. Teghtsoonian, The standard model for perceived magnitude: A framework for (almost) everything known about it, Am. J. Psychol. 125, 165 (2012).

[7] D. E. Kornbrot, Human psychophysical functions, an update: Methods for identifying their form; estimating their parameters; and evaluating the effects of important predictors, Psychometrika 81, 201 (2016).

[8] J. M. Beggs and D. Plenz, Neuronal avalanches in neocortical circuits, J. Neurosci. 23, 11167 (2003).

[9] J. M. Beggs, The criticality hypothesis: How local cortical networks might optimize information processing, Philos. Trans. R. Soc. A 366, 329 (2008).

[10] D. R. Chialvo, Emergent complex neural dynamics, Nat. Phys. 6, 744 (2010).

[11] W. L. Shew and D. Plenz, The functional benefits of criticality in the cortex, Neuroscientist 19, 88 (2013).

[12] M. A. Munoz, Colloquium: Criticality and dynamical scaling in living systems, Rev. Mod. Phys. 90, 031001 (2018).

[13] J. Wilting and V. Priesemann, 25 years of criticality in neuroscience-established results, open controversies, novel concepts, Curr. Opin. Neurobiol. 58, 105 (2019).

[14] O. Kinouchi and M. Copelli, Optimal dynamical range of excitable networks at criticality, Nat. Phys. 2, 348 (2006).

[15] M. Copelli, Physics of psychophysics: It is critical to sense, in Cooperative Behavior in Neural Systems: Ninth Granada Lectures, edited by J. Marro, P. L. Garrido, and J. J. Torres, AIP Conf. Proc. No. 887 (AIP, New York, 2007), p. 13.

[16] V. R. V. Assis and M. Copelli, Dynamic range of hypercubic stochastic excitable media, Phys. Rev. E 77, 011923 (2008).

[17] W. L. Shew, H. Yang, T. Petermann, R. Roy, and D. Plenz, Neuronal avalanches imply maximum dynamic range in cortical networks at criticality, J. Neurosci. 29, 15595 (2009).

[18] D. B. Larremore, W. L. Shew, and J. G. Restrepo, Predicting Criticality and Dynamic Range in Complex Networks: Effects of Topology, Phys. Rev. Lett. 106, 058101 (2011).

[19] S. Pei, S. Tang, S. Yan, S. Jiang, X. Zhang, and Z. Zheng, How to enhance the dynamic range of excitatory-inhibitory excitable networks, Phys. Rev. E 86, 021909 (2012).

[20] C. A. S. Batista, R. L. Viana, S. R. Lopes, and A. M. Batista, Dynamic range in small-world networks of Hodgkin-Huxley neurons with chemical synapses, Physica A 410, 628 (2014).

[21] C.-Y. Wang, Z.-X. Wu, and M. Z. Q. Chen, Approximatemaster-equation approach for the Kinouchi-Copelli neural model on networks, Phys. Rev. E 95, 012310 (2017).

[22] J. G. F. Campos, A. A. Costa, M. Copelli, and O. Kinouchi, Correlations induced by depressing synapses in critically selforganized networks with quenched dynamics, Phys. Rev. E 95, 042303 (2017).

[23] R. Zhang and S. Pei, Dynamic range maximization in excitable networks, Chaos 28, 013103 (2018).

[24] S. H. Gautam, T. T. Hoang, K. McClanahan, S. K. Grady, and W. L. Shew, Maximizing sensory dynamic range by tuning the cortical state to criticality, PLOS Comput. Biol. 11, e1004576 (2015).
[25] W. L. Shew, W. P. Clawson, J. Pobst, Y. Karimipanah, N. C. Wright, and R. Wessel, Adaptation to sensory input tunes visual cortex to criticality, Nat. Phys. 11, 659 (2015).

[26] C. G. Antonopoulos, Dynamic range in the C. elegans brain network, Chaos 26, 013102 (2016).

[27] M. Copelli, A. C. Roque, R. F. Oliveira, and O. Kinouchi, Physics of psychophysics: Stevens and Weber-Fechner laws are transfer functions of excitable media, Phys. Rev. E 65, 060901 (2002).

[28] M. Copelli and O. Kinouchi, Intensity coding in twodimensional excitable neural networks, Physica A 349, 431 (2005).

[29] M. Copelli, R. F. Oliveira, A. C. Roque, and O. Kinouchi, Signal compression in the sensory periphery, Neurocomputing 65, 691 (2005).

[30] L. L. Gollo, O. Kinouchi, and M. Copelli, Active dendrites enhance neuronal dynamic range, PLOS Comput. Biol. 5, e1000402 (2009).

[31] T. A. Cleland and C. Linster, Concentration tuning mediated by spare receptor capacity in olfactory sensory neurons: A theoretical study, Neural Comput. 11, 1673 (1999).

[32] T. A. Cleland and C. Linster, Computation in the olfactory system, Chem. Senses 30, 801 (2005).

[33] H.-K. Janssen, On the nonequilibrium phase transition in reaction-diffusion systems with an absorbing stationary state, Z. Phys. B 42, 151 (1981).

[34] P. Grassberger, On phase transitions in Schlögl's second model, Z. Physik B - Condensed Matter 47, 365 (1982).

[35] L. Brochini, A. A. Costa, M. Abadi, A. C. Roque, J. Stolfi, and $\mathrm{O}$. Kinouchi, Phase transitions and self-organized criticality in networks of stochastic spiking neurons, Sci. Rep. 6, 35831 (2016).

[36] M. Girardi-Schappo, L. Brochini, A. A. Costa, T. T. A. Carvalho, and O. Kinouchi, Synaptic balance due to homeostatically self-organized quasicritical dynamics, Phys. Rev. Res. 2, 012042(R) (2020).

[37] L. A. B. Martinez and M. H. R. Tragtenberg, Enhancement of the dynamic range in interconnected layered random networks (private communication).

[38] W. Gerstner and J. L. van Hemmen, Associative memory in a network of "spiking" neurons, Netw. Comput. Neural Syst. 3, 139 (1992).

[39] A. Galves and E. Löcherbach, Infinite systems of interacting chains with memory of variable length-A stochastic model for biological neural nets, J. Stat. Phys. 151, 896 (2013).

[40] P. A. Ferrari, A. Galves, I. Grigorescu, and E. Löcherbach, Phase transition for infinite systems of spiking neurons, J. Stat. Phys. 172, 1564 (2018).

[41] A. A. Costa, L. Brochini, and O. Kinouchi, Self-organized supercriticality and oscillations in networks of stochastic spiking neurons, Entropy 19, 399 (2017).

[42] O. Kinouchi, L. Brochini, A. A. Costa, J. G. F. Campos, and M. Copelli, Stochastic oscillations and dragon king avalanches in self-organized quasicritical systems, Sci. Rep. 9, 3874 (2019).

[43] B. Cessac, A discrete time neural network model with spiking neurons, J. Math. Biol. 56, 311 (2008). 
[44] B. Cessac, A view of neural networks as dynamical systems, Int. J. Bifurc. Chaos Appl. Sci. Eng. 20, 1585 (2010).

[45] B. Cessac, A discrete time neural network model with spiking neurons: II. Dynamics with noise, J. Math. Biol. 62, 863 (2011).

[46] J. Benda and A. V. M. Herz, A universal model for spike-frequency adaptation, Neural Comput. 15, 2523 (2003).

[47] A. Buonocore, L. Caputo, E. Pirozzi, and M. F. Carfora, A leaky integrate-and-fire model with adaptation for the generation of a spike train, Math. Biosci. Eng. 13, 483 (2016).

[48] H. Hinrichsen, Nonequilibrium critical phenomena and phase transitions into absorbing states, Adv. Phys. 49, 815 (2000).

[49] S. Lübeck, Universal scaling behavior of nonequilibrium phase transitions, Int. J. Mod. Phys. B 18, 3977 (2004).

[50] M. A. Muñoz, R. Dickman, A. Vespignani, and S. Zapperi, Avalanche and spreading exponents in systems with absorbing states, Phys. Rev. E 59, 6175 (1999).

[51] A. A. Costa, M. Copelli, and O. Kinouchi, Can dynamical synapses produce true self-organized criticality? J. Stat. Mech.: Theory Expt. (2015) P06004.

[52] J. A. Bonachela and M. A. Muñoz, Self-organization without conservation: True or just apparent scale invariance? J. Stat. Mech.: Theory Expt. (2009) P09009.
[53] J. A. Bonachela, S. deFranciscis, J. J. Torres, and M. A. Muñoz, Self-organization without conservation: Are neuronal avalanches generically critical? J. Stat. Mech.: Theory Expt. (2010) P02015.

[54] A. Levina, J. M. Herrmann, and T. Geisel, Dynamical synapses causing self-organized criticality in neural networks, Nat. Phys. 3, 857 (2007).

[55] J. E. Cook and D. L. Becker, Gap junctions in the vertebrate retina, Microsc. Res. Tech. 31, 408 (1995).

[56] J. O'Brien, The ever-changing electrical synapse, Curr. Opin. Neurobiol. 29, 64 (2014).

[57] M. R. Deans, B. Volgyi, D. A. Goodenough, S. A. Bloomfield, and D. L. Paul, Connexin36 is essential for transmission of rodmediated visual signals in the mammalian retina, Neuron $\mathbf{3 6}$, 703 (2002).

[58] G. J. Murphy and F. Rieke, Electrical synaptic input to ganglion cells underlies differences in the output and absolute sensitivity of parallel retinal circuits, J. Neurosci. 31, 12218 (2011).

[59] R. Publio, R. F. Oliveira, and A. C. Roque, A computational study on the role of gap junctions and rod ih conductance in the enhancement of the dynamic range of the retina, PLoS One 4, e6970 (2009).

[60] R. Pazzini and O. Kinouchi, Neuronal avalanches in WattsStrogatts networks of stochastic spiking neurons (private communication). 